\title{
Frequently asked questions of individuals with spinal cord injuries: results of a web-based consultation service in Iran
}

\author{
Mojtaba Rezaei ${ }^{1} \cdot$ Mahmoud Omidbeigi $^{2} \cdot$ Sara Hanaei ${ }^{3,4} \cdot$ Negin Saeedi $^{5} \cdot$ Khatereh Naghdi $^{1}$ • \\ Alexander R. Vaccaro $^{6} \cdot$ Vafa Rahimi-Movaghar $^{1,7,8,9}$
}

Received: 16 December 2017 / Revised: 31 March 2018 / Accepted: 5 April 2018

(C) International Spinal Cord Society 2018

\begin{abstract}
Study design Descriptive study of the results of a web-based consultation service for individuals with spinal cord injury (SCI).

Objectives To review frequently asked questions (FAQ) among individuals with SCI and determine the most important topics.

Setting Sina Trauma and Surgery Research Center, Tehran University of Medical Sciences, Tehran, Iran.

Methods A cross-sectional study reviewing questions from patients with SCI that were collected from the Health and Safety Consultant Center (HSCC) between January and December 2015. The HSCC is a web-based medical consultation service that provides patients with information and counseling pertaining to SCI.

Results A total of 113 questions were collected from 99 individuals. The mean age was $32.02 \pm 13.28$ years with a range of 3-70 years. Men accounted for $81.7 \%$ of the questions, while women accounted for $18.3 \%$. The most common site of SCI was thoracic $(40.6 \%)$, followed by lumbar $(31.3 \%)$, and cervical $(28.1 \%)$. Recovery potential $(38.1 \%)$, sexual and reproductive issues $(26.5 \%)$, urinary $(10.6 \%)$, and motor $(10.6 \%)$ problems were among the most commonly reviewed topics. Conclusions FAQ provide insight on areas of concerns for individuals with SCI and help guide providers to determine highyield topics. Discerning the specific areas of need or concern for patients is instrumental in developing pertinent educational materials and programs, in addition to efficiently counseling patients and caregivers on the aftercare of SCI.
\end{abstract}

\section{Introduction}

A spinal cord injury (SCI) is a life-altering experience that results in significant physical and mental morbidity with lifelong implications [1]. In addition, individuals with SCI and their caregivers face many challenges leaving them with

Vafa Rahimi-Movaghar

v_rahimi@sina.tums.ac.ir

v_rahimi@yahoo.com

1 Sina Trauma and Surgery Research Center, Tehran University of Medical Sciences, Tehran, Iran

2 Clinical Research Development Center, Loghman Hakim Hospital, Shahid Beheshti University of Medical Sciences, Tehran, Iran

3 School of Medicine, Tehran University of Medical Sciences (TUMS), Tehran, Iran

4 Universal Scientific Education and Research Network (USERN), Tehran, Iran numerous questions about the extent of their disability, lifestyle modifications, and potential complications. Providing patients and caregivers with adequate and relevant information has been shown to improve medical management of chronic disorders and reduce hospital admissions [2]. 
Studies have shown that patients are often dissatisfied with the level of communication with their physicians [3, 4]. Often this is a result of differences between the physician's judgment and the patient's preference [5]. Therefore, it is vital for providers who partake in the care of individuals with SCI to be familiar with frequently asked questions (FAQ) in order to provide them with relevant and focused answers, in addition to improving counseling.

Other educational materials such as brochures, booklets, websites, web-based consultation services, and peer-support groups are also available, each one having its own strengths and weaknesses [4, 6, 7]. Online references have become a major source of information for patients [6, 8]. However, gathering information from numerous online sources with variable reliability makes it difficult for patients to obtain accurate information. Therefore, a web-based consultation service is a potential way to reduce this problem [9].

While various FAQ resources pertaining to SCI are available, they have been predominantly generated by medical professionals. However, these resources can be made more effective when delivered in a patient-centered and user-friendly manner. Providers should be aware of these FAQ in order to provide thorough and easy-tounderstand responses. The literature supports the positive effects of a list of FAQ on the quality of communication between patient and physician $[10,11]$. However, these studies did not develop a comprehensive list of FAQ based on a high level of evidence. In addition, to the best of our knowledge, a list of evidence-based FAQ obtained from individuals with SCI has not been developed. The aim of this study was to ascertain questions asked by SCI patients in order to better define areas of concern and to establish a list of FAQ and create an informative online resource for individuals with SCI.

\section{Methods}

The Health and Safety Consultant Center developed a web-based medical consultation service (www.hscc.ir) in 2013 to provide information and answers about various SCI-related topics. All incoming questions between January and December 2015 were analyzed. To provide a more individualized answer, the web-based application first controlled for demographics including age, gender, mechanism, and site of injury. A neurosurgeon (VRM) was then responsible for providing answers utilizing the most current evidence. Any ethical or legal question or those that could not be thoroughly answered through the service were referred to a clinic. No medications were prescribed through this service. The consultation was free of charge and the consulting physicians were not monetarily compensated. Submitted questions and answers were

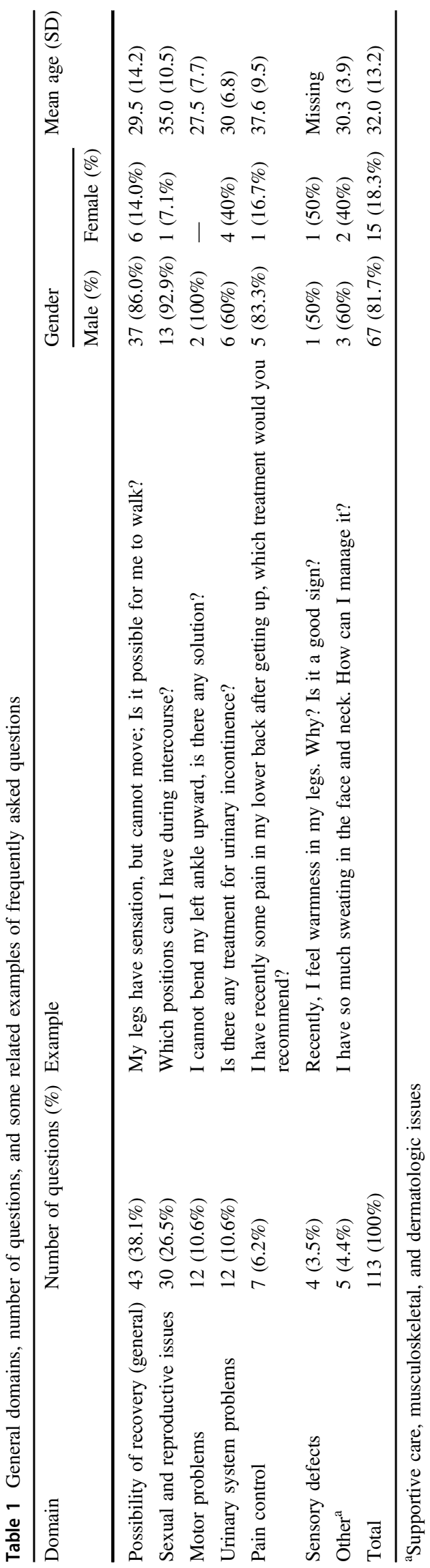


available online for public viewing. Questions were allowed to be asked in any language, however all answers were provided in Persian.

\section{Results}

A total of 113 questions were collected from 99 patients with SCI. On average, participants were $32.02 \pm 13.28$ years of age with a range of 3-70 years. Of the questions submitted, $81.7 \%$ were from men and $18.3 \%$ were from women. The most common site of SCI was thoracic (40.6\%), followed by lumbar (31.3\%), and cervical $(28.1 \%)$. Trauma was the main cause of injury accounting for $56(82.3 \%)$ cases which were further subdivided into car accidents $(n=34,50 \%)$, fall $(n=12,21.4 \%)$ and other types of trauma $(n=10,14.7 \%)$. Other causes of SCI accounted for $17.7 \%(n=12)$ patients.

Table 1 shows the different health domains that each question falls into, the distribution within each domain, and some related examples. Possibility of recovery (38.1\%), sexual and reproductive issues (26.5\%), urinary (10.6\%), and motor $(10.6 \%)$ problems were among the most commonly discussed topics. Most sexual and reproductive related questions were raised by men (male 92.9\%; female 7.1\%). Men were concerned with erectile dysfunction, anejaculation, and lack of penile sensation during intercourse. Women were mostly concerned with reproductivity, specifically about prenatal care and choosing the best method for childbirth delivery (Table 1).

\section{Discussion}

Spinal cord injuries place a large burden on patients, their families, caregivers, health systems, and society. The enduring consequences of SCI leave patients with many questions and concerns. Identifying these areas of importance can help providers better educate patients on the aftercare following a SCI. Based on a literature review, this is the first study to provide an evidence-based list of FAQ from individuals with SCI.

Over a 1-year period, 99 participants asked 113 questions through a web-based service. The number of website visits and questions asked were lower than expected, which may be due to a lack of public awareness about the consultation service. In addition, the availability of questions and answers for public viewing may provide the needed information for others and therefore limit the number of consultations. Individuals with SCI often use the internet for interpersonal communication and to access health and disability-related information, and have shown a significant benefit in health-related quality of life among internet users
[12]. Therefore, a web-based consultation service can be implemented on a larger scale to provide patients with reliable information and ultimately enhance patient care for those with SCI.

The age range for participants was 3-70 years of age. The wide range may be representative of questions raised by the parents or caregivers of patients at both ends of the spectrum. However, it should be noted that most of the questions were posed by participants between the ages of 16 and 30 years of age. Among participants, $81.7 \%$ were male and only $18.3 \%$ were female, which is consistent with gender distribution of SCI [1]. In addition, trauma accounted for $82.3 \%$ of SCI mechanisms that are also consistent with prior literature [1].

Recovery potential was the most FAQ among individuals with SCI in this group. Our findings also demonstrated that even after several years of living with and managing a SCI, patients continued to have ongoing concerns pertaining to their care. Since the response to this type of question is highly dependent on various patient factors, it is necessary to individually address these concerns.

Questions concerning sexual and reproductive issues were the next most commonly discussed topics. Asking questions related to sexual issues may be very difficult during face to face visits in some cultures, therefore, it is recommended that health care providers actively ask patients about this problem.

One of the strengths of this study is that individuals had the freedom to ask sensitive questions. In addition, due to the time constraints for physicians during office visits this can lead to questions being left unanswered. In contrast, a web-based application allows patients to engage physicians with thoughtful questions, as well as, allow physicians the time to construct a well-thought-out answer with evidencebased references. Also, this application increases access and timeliness of delivery of quality care while reducing geographic barriers.

A limitation of this study is the lack of accessibility to broadband connectivity and computer illiteracy for some patients, in particular for the elderly and underserved populations [13]. Access to the internet is also limited in suburb and some urban areas. Consequently, nonresponders may not be adequately represented by participants, resulting in participation bias and limiting the generalizability of these results.

We identified areas of concern for patients with SCI, which will help providers develop patient-specific educational materials and programs. Having a FAQ list available can help health care provider caring for this unique population to be prepared for answering questions based on the best evidence available and to actively address concerns early on. 


\section{Conclusion}

FAQ provide both valuable insight on SCI and help providers determine common topics that need further clarification. Recovery potential and sexual and reproductive issues are among the most important issues for patients who suffered a SCI. According to prior studies [12, 14, 15], motor difficulties, urinary problems, and recovery potential are issues that have a significant impact on quality of life and may explain why these are among the most FAQ for individuals with SCI.

Discerning these specific areas of need or concern for patients is instrumental in developing pertinent educational materials and programs, in addition to efficiently counseling patients and caregivers on the aftercare of SCI.

Acknowledgements This research has been supported by Tehran University of Medical Sciences and Health Services.

\section{Compliance with ethical standards}

Conflict of interest ARV is a board member of AOSpine, Innovative Surgical Design, Association of Collaborative Spine Research, DePuy; Consultant at Medtronics, Stryker Spine, Globus, Stout Medical, Gerson Lehrman Group, Guidepoint Global, Medacorp, Innovative Surgical Design, Orthobullets, Ellipse, Vertex, Medtronics; Royalty at Stryker Spine, Biomet Spine, Globus, Aesculap, Thieme, Jaypee, Elsevier, Taylor Francis. All the remaining authors declare that they have no conflict of interest.

\section{References}

1. Rahimi-Movaghar V, Sayyah MK, Akbari H, et al. Epidemiology of traumatic spinal cord injury in developing countries: a systematic review. Neuroepidemiology. 2013;41:65-85.

2. Panagioti M, Richardson G, Murray E, et al. Reducing care utilisation through self-management interventions (RECURSIVE): a systematic review and meta-analysis. Southampton, UK: NIHR Journals Library; 2014.

3. Chaitchik S, Kreitler S, Shaked S, Schwartz I, Rosin R. Doctorpatient communication in a cancer ward. J Cancer Educ. 1992;7:41-54.

4. Norman C, Bender JL, Macdonald J, et al. Questions that individuals with spinal cord injury have regarding their chronic pain: a qualitative study. Disabil Rehabil. 2010;32:114-24.

5. Whitehurst DG, Noonan VK, Dvorak MF, Bryan S. A review of preference-based health-related quality of life questionnaires in spinal cord injury research. Spinal Cord. 2012;50:646-54.

6. Tuffrey C, Finlay F. Use of the internet by parents of paediatric outpatients. Arch Dis Child. 2002;87:534-6.

7. Whitford DL, Paul G, Smith SM. Patient generated "frequently asked questions": Identifying informational needs in a RCT of peer support in type 2 diabetes. Prim Care Diabetes. 2013;7:103-9.

8. Goodman N, Jette AM, Houlihan B, Williams S. Computer and internet use by persons after traumatic spinal cord injury. Arch Phys Med Rehabil. 2008;89:1492-8.

9. Kruisinga FH, Heinen RC, Heymans HS. Analysis of the question-answer service of the Emma Children's Hospital information centre. Eur J Pediatr. 2010;169:853-60.

10. Bolman C, Brug J, Bar F, Martinali J, van den Borne B. Longterm efficacy of a checklist to improve patient education in cardiology. Patient Educ Couns. 2005;56:240-8.

11. Grant RW, Altschuler A, Uratsu CS, et al. Primary care visit preparation and communication for patients with poorly controlled diabetes: a qualitative study of patients and physicians. Prim Care Diabetes. 2017;11:148-53.

12. Drainoni M-L, Houlihan B, Williams S, et al. Patterns of Internet use by persons with spinal cord injuries and relationship to healthrelated quality of life. Arch Phys Med Rehabil. 2004;85:1872-9.

13. Carlson BA, Neal D, Magwood G, Jenkins C, King MG, Hossler CL. A community-based participatory health information needs assessment to help eliminate diabetes information disparities. Health Promot Pract. 2006;7(3_suppl):213S-22S.

14. Hill MR, Noonan VK, Sakakibara BM, Miller WC, Team SR. Quality of life instruments and definitions in individuals with spinal cord injury: a systematic review. Spinal Cord. 2010;48:438.

15. Munce SE, Perrier L, Tricco AC, et al. Impact of quality improvement strategies on the quality of life and well-being of individuals with spinal cord injury: a systematic review protocol. Syst Rev. 2013;2:14. 